\title{
Non-anatomical polymer phantoms: a new tool to develop and assess technical skills in ultrasound guided procedures
}

\author{
Jan-Alexis Tremblay, François Marquis
}

Intensive Care Unit, Maisonneuve-Rosemont Hospital, Montréal, Canada.

\section{To the Editor}

Ultrasound (US) can reduce the number of punctures and complication rates of several types of vascular cannulation without lengthening the duration of the intervention [1]. However, even with US, the rate of posterior wall penetration during jugular vein catheterization, for instance, can exceed $60 \%$ when performed by junior residents [2]. Learning US guided procedures is still achieved mainly in the clinical setting: not only this exposes patients to real risks, but time constraints may also limit the acquisition of spatial orientation and handeye coordination [3]. In this perspective, a consensus is emerging on the need to develop simulation-based learning curricula for ultrasound guided procedures [4].

Synthetic anatomical models called "phantoms" whose sonographic appearance is very close to human tissue have been commercially available for years, but the use of commercial phantoms is limited by their high cost. Conversely, home-made phantoms fashioned from various materials (animal tissues, gelatins, agar, tofu) have been described but tend to be fragile and have a limited lifetime [5]. We therefore aimed to manufacture a phantom which is durable, safe, acoustically satisfying and inexpensive using polymeric materials.

We thus present a new type of silicone phantom designed to improve spatial orientation and hand-eye coordination in US guided procedures in a play-based format. Phantoms were made out of platinum-based silicones (Smooth-On Inc. Easton, PN, USA). For the core of the

Received 02.02.2016 Accepted 5.03.2016

Med Ultrason

2016, Vol. 18, No 2, 260-261, DOI: 10.11152/mu.2013.2066.182.ply

Corresponding author: Jan-Alexis Tremblay

5415 Boulevard de l'Assomption,

Montreal, Quebec, Canada.

H1T 2M4

Phone: (514) 252-3400, (514) 347-0163

Fax: 18665281826

E-mail: janalexis.tremblay@gmail.com phantom, we selected a gel-like silicone of almost liquid consistency, giving it self-healing properties after needle passage. The walls and top of the phantom were made out of a stronger skin-like silicone, as were the targets inside the phantom. For these, to allow electrical conductivity through the silicone while retaining flexibility, carbon fiber (commercially available as woven fabric, then cut into 10 $\mathrm{mm}$ fiber length) was added at $2 \%$ of the total weight of the mixture. As they are easily recognizable by US, wellknown plastic toy figures (Playmobil Inc., Geobra Brandstätter GmbH \& Co. KG, Zirndorf, Germany) were used after being coated with the silicon-carbon fibre mixture. Silicone compatible pigments from the same company

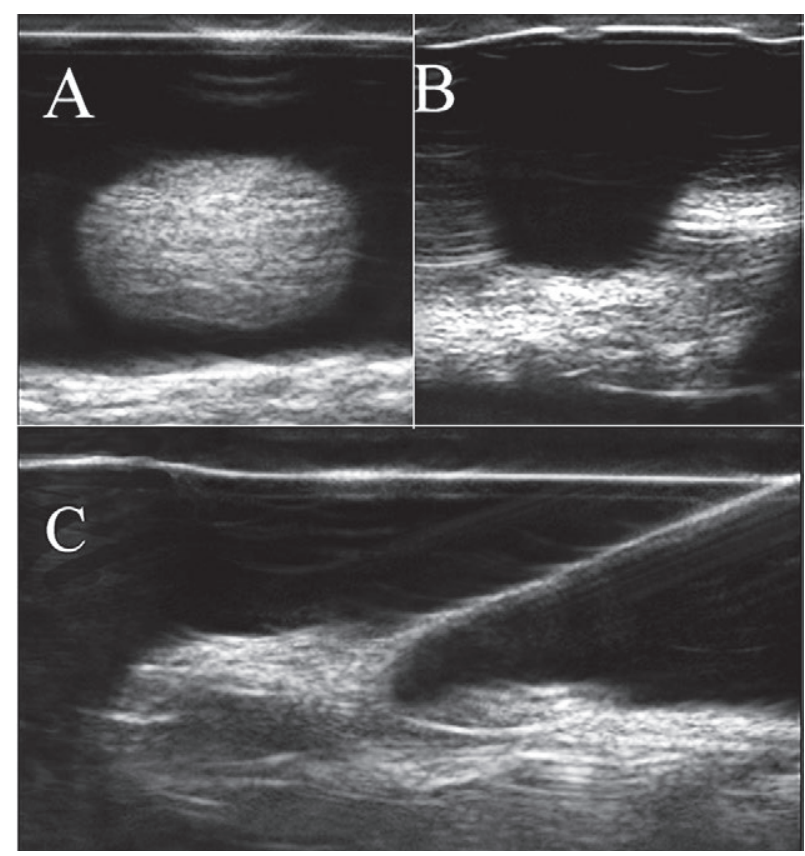

Fig 1. Ultrasonographic rendering of silicone phantoms. A: Hyperechoic spherical target. B: Plastic toy figure torso with both arms outreaching to the ultrasound probe. C: Plastic toy figure in dorsal decubitus, with the needle contacting its head. (Images from SonoSite Edge ultrasound machine. Fujifilm SonoSite. Bothell, Washington, USA) 
were added in the mixing step to ensure visual opacity of the phantoms. Degassing with a vacuum chamber at -29 inHg was done after each pouring to remove entrapped bubbles. After curing, phantoms were connected to a simple analog circuit allowing confirmation of the contact of the needle with red and green LED.

Total monetary cost for each of our phantoms was between 15 and 20 \$US. Actual hands-on working time (excluding curing) was about one hour for each phantom. The core of the phantom was found to be self-healing, with complete acoustical obliteration of needle tracks after about 15 minutes. One year of use later, the gel-like silicon still self-heals, although the skin of the phantom shows the numerous puncture sites from which there is occasional leakage of the gel-like core.

Finally, even though the increased acoustical attenuation of silicone warrants high gain levels and somehow reduce image quality compared to human tissue (Figure 1), we found that it did not really hamper the usefulness of the phantom as a learning tool. Indeed, this has to be seen as an introduction to ultrasound probe manipulation and as a first step for learners in acquiring hand-eye coordination and spatial recognition in ultrasound procedures, before moving to more costly and fragile anatomical phantoms and eventually actual human patients, as these procedures require technical skills and spatial recognition that can only emerge by dint of repetition and practice.

Acknowledgments: To the residents of Maisonneuve-Rosemont hospital for testing the phantom and for providing us with precious insight.

\section{References}

1. Airapetian N, Maizel J, Langelle F, et al. Ultrasound-guided central venous cannulation is superior to quick-look ultrasound and landmark methods among inexperienced operators: A prospective randomized study. Intensive Care Med 2013; 39: 1938-1944

2. Blaivas M, Adhikari S. An unseen danger: Frequency of posterior vessel wall penetration by needles during attempts to place internal jugular vein central catheters using ultrasound guidance. Critic Care Med 2009; 37: 2345-2349; quiz 2359

3. Adhikary SD, Hadzic A, McQuillan PM. Simulator for teaching hand-eye coordination during ultrasound-guided regional anaesthesia. BrJ Anesth 2013; 111: 844-845

4. Farjad Sultan S, Shorten G, Iohom G. Simulators for training in ultrasound guided procedures. Med Ultrason 2013; 15: $125-131$

5. Culjat MO, Goldenberg D, Tewari P, Singh RS. A review of tissue substitutes for ultrasound imaging. Ultrasound Med Biol 2010; 36: 861-873

\title{
A very rare cause of recurrent back pain: hematocolpometra
}

\section{Zeynep Ozcan Dag¹, Ozlem Banu Tulmac¹, Yuksel Isik}

\author{
${ }^{1}$ Department of Obstetrics and Gynecology, Faculty of Medicine, Kirikkale University, Kirikkale, Turkey
}

\section{To the Editor,}

A 15 year old girl was admitted to with recurrent back pain about four or five times last year. Her medical history was unremarkable. She reported that she had never been sexually active and in her gynecological history there was no vaginal discharge or menstruation. On physical examina-

Received 23.03.2016 Accepted 06.04.2016

Med Ultrason

2016, Vol. 18, No 2, 261-262, DOI: 10.11152/mu.2013.2066.182.zey

Corresponding author: Zeynep Ozcan Dag

Kirikkale University, Faculty of Medicine, Department of Obstetrics and Gynecology, 71300 Kirikkale, Turkey

Phone: +90 05326080725, Fax: +90 3182240786

E-mail: zozcan23@yahoo.com tion a palpable, nontender midline mass from the pelvis to the umblicus was revealed. After abdominal ultrasound was done she was referred to our clinic with a preliminary diagnosis of ovarian mucinous cystadenoma $11 \times 35 \mathrm{~cm}$ in size. Pelvic ultrasonography revealed hematocolpometra with a dilated vagina measuring $23.7 \mathrm{~cm}$ in length, $9.5 \mathrm{~cm}$ in width, and $8 \mathrm{~cm}$ in height and an uterus $6,5 \mathrm{~cm}$ in length, $4.3 \mathrm{~cm}$ in width, $4.2 \mathrm{~cm}$ in height which was filled up with hypoechoic homogenous fluid (fig 1). Pelvic examination revealed a bulging bluish imperforate hymen. She was taken to the operating room next morning. Stellate incisions were made through the hymenal membrane at the 2, 4, 8, and 10 o'clock positions under general anesthesia. The edges were excised and sutured with fine delayed absorbable suture. Approximately 2200 cc dark, thick blood was drained. At the fol- 


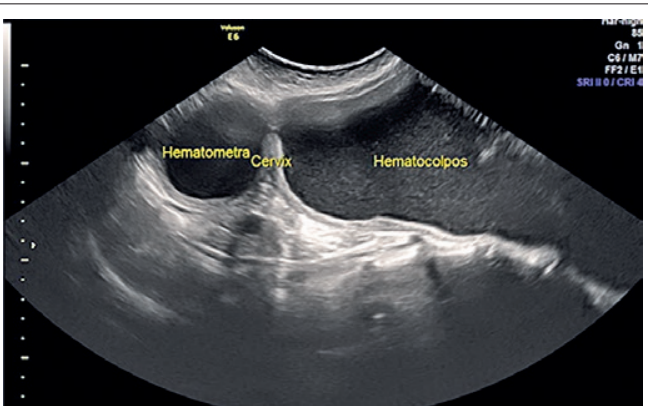

Fig 1. Ultrasound scan showing the fluid in the vagina (hematocolpos) and showing fluid-filled uterus (hematometra).

lowing visits 2 weeks later and 2 months later she described a normal menstrual period and ultrasonography revealed a normal endometrium and uterus with a thick and long cervix.

Children with imperforate hymen usually remain asymptomatic until menarche. They are admitted to the gynecologists at about 13-15 years with primary amenorrhea and the symptoms appear due to accumulation of blood in the vagina. The most common symptoms are cyclic abdominal pain, pelvic discomfort, chronic constipation, acute urinary retention, urinary tract infections and low back pain [1]. The delay in diagnosis leads to increased morbidity and mortality, including laparotomy, because of suspected malignancy, respiratory failure sec- ondary to abdominal distension, bowel perforation and some sexual dysfunction such as menstrual irregularities, endometriosis and infertility $[2,3]$. Therefore, early diagnosis and timely of the treatment is great importance.

Imperforate hymen should be considered in premenarcheal girls with back pain in the presence of primary amenorrhea. Despite the ease diagnosis at all ages with pelvic examination, many clinicians are reluctant to do the pelvic examination, so that the diagnosis of imperforate hymen is delayed or missed resulting in preventable morbidity such as endometriosis, peritonitis due to the retrograde flow and hydronephrosis due to the compression. On pelvic examination a bulging bluish hymenal membrane confirms the diagnosis of imperforate hymen and a pelvic abdominal ultrasound shows the pelvic cystic mass.

\section{References}

1. Posner JC, Spandorfer PR. Early detection of imperforate hymen prevents morbidity from delays in diagnosis. Pediatrics 2005; 115: 1008-1012.

2. Shaw LM, Jones WA, Brereton RJ. Imperforate hymen and vaginal atresia and their associated anomalies. J R Soc Med 1983; 76: 560-566.

3. Nazir Z, Rizvi RM, Qureshi RN, Khan ZS, Khan Z. Congenital vaginal obstructions: varied presentation and outcome. Pediatr Surg Int 2006; 22: 749-753.

\title{
Successful management of a second trimester cesarean scar pregnancy using local methotrexate injection
}

\author{
Vincent Y.T. Cheung, Tsz-kin Lo
}

Department of Obstetrics and Gynaecology, Queen Mary Hospital, The University of Hong Kong, Hong Kong

\section{To the Editor,}

A 37-year-old woman, gravida 6 para 2, was referred for pregnancy termination at 13 weeks' gestation. She had 2 previous cesarean deliveries and 3 pregnancy ter-

Received Accepted

Med Ultrason

2016, Vol. 18, No 2,262-263, DOI: 10.11152/mu.2013.2066.182.mth

Corresponding author: Vincent Y.T. Cheung

Department of Obstetrics and Gynaecology, Queen Mary Hospital,

102 Pokfulam Road, Hong Kong

Phone: 852-22553914, Fax: 852-25173278

E-mail: vytc@hku.hk minations. Pelvic examination showed a 14 week-sized uterus. Ultrasound showed a 14-week cesarean scar pregnancy (CSP) with cardiac pulsation evident (fig 1). Serum human chorionic gonadotropin (hCG) level was 78822 IU/L. After counselling on the significance of her condition and treatment options, local methotrexate injection, the first-line treatment for CSP in our institution [1,2], was decided. Under transabdominal ultrasound guidance, a 20-gauge spinal needle was inserted into the fetal heart, through which $2 \mathrm{ml}$ of $14.9 \%$ potassium chloride was injected. Following cessation of fetal heart pulsation, 60 $\mathrm{ml}$ of amniotic fluid was withdrawn and $25 \mathrm{mg}$ of methotrexate was injected intra-amniotically. Her hCG levels 


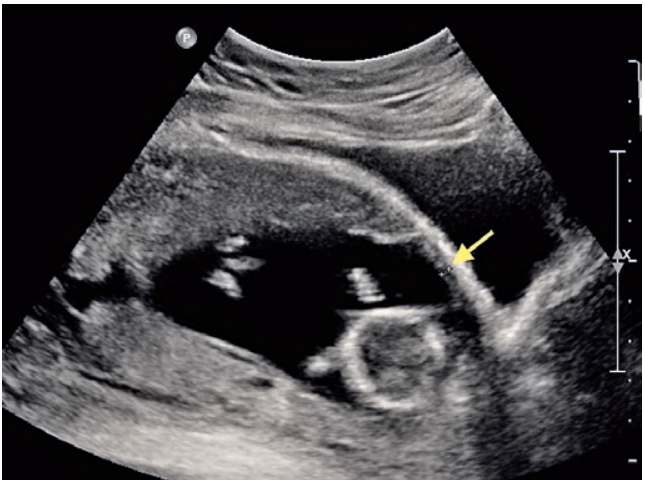

Fig 1. Ultrasound showing a gestational sac containing a single fetus with cardiac pulsation evident. Empty uterine cavity and focal thinning of the overlying myometrium (arrow, $0.2 \mathrm{~cm}$ ) were noted. Fetal biometry: crown-rump length $=7.26 \mathrm{~cm}(13.5$ weeks); biparietal diameter $=2.57 \mathrm{~cm}(14.5$ weeks $)$.

dropped to $63001 \mathrm{IU} / \mathrm{L}$ and $35736 \mathrm{IU} / \mathrm{L}$ on the third and seventh post-injection day respectively, and normalized after 12 weeks. She had an episode of moderate vaginal bleeding 8 weeks after the injection followed by regular menses. Ultrasound showed continual regression in size of the gestational mass to $1-2 \mathrm{~cm}$ after 10 months.

To our knowledge, successful management of second trimester CSP using local methotrexate injection has never been reported. Currently, for CSP with no or minimal symptom, there is no consensus on the optimal management. CSP, if left untreated, is also consider a precursor of morbidly adherent placenta, and carries a substantial risk of uterine rupture and the need for hysterectomy $[3,4]$. The management of "advanced" CSP clearly poses a much greater challenge because of its higher risk of complications [5]. A review of literature obtained from PubMed database from inception to December 2015 using the keywords "cesarean/caesarean scar", "ectopic pregnancy" and "second trimester" or "advanced", only identified 2 cases of relatively asymptomatic second trimester CSP. One 31-year-old woman presented with spotting at 13-week, underwent suction curettage for CSP; ultimately required supracervical hysterectomy for bleeding control [6]. Another 21-year-old woman at 13.5-week, had surgical removal of CSP via laparotomy; also resulted in supracervical hysterectomy [7]. In another review, 20 of 751 cases of CSP were between 10 and 15 weeks' gestation, but treatment details were not presented [5]. As local methotrexate injection has a low complication rate [5], we suggest it can be considered an option in the management of second trimester CSP. Together with the understanding that a single case report cannot be generalized, we also advocate that all unusual or advanced CSP should be reported so that more information on safety and efficacy of various treatment modalities can be collected.

\section{References}

1. Cheung VY. Local Methotrexate Injection as the First-line Treatment for Cesarean Scar Pregnancy: Review of the Literature. J Minim Invasive Gynecol 2015; 22: 753-758.

2. Ko JK, Li RH, Cheung VY. Caesarean scar pregnancy: a 10-year experience. Aust N Z J Obstet Gynaecol 2015; 55: 64-69.

3. Michaels AY, Washburn EE, Pocius KD, Benson CB, Doubilet PM, Carusi DA. Outcome of cesarean scar pregnancies diagnosed sonographically in the first trimester. J U1trasound Med 2015; 34: 595-599.

4. Timor-Tritsch IE, Khatib N, Monteagudo A, Ramos J, Berg R, Kovács S. Cesarean scar pregnancies: experience of 60 cases. J Ultrasound Med 2015; 34: 601-610.

5. Timor-Tritsch IE, Monteagudo A. Unforeseen consequences of the increasing rate of cesarean deliveries: early placenta accreta and cesarean scar pregnancy. A review. Am J Obstet Gynecol 2012; 207: 14-29.

6. Dickerhoff LA, Mahal AS, Stockdale CK, Hardy-Fairbanks AJ. Management of cesarean scar pregnancy in the second trimester: a report of three cases. J Reprod Med 2015; 60): 165-168.

7. Overcash RT, Khackician ZH. Late-first-trimester cesarean section scar ectopic pregnancy with placenta increta: a case report. J Reprod Med 2012; 57: 61-64. 


\title{
Comment on "The use of Doppler ultrasonography for pre- and post- surgery monitoring of abdominal aortic aneurysm. A case report".
}

\author{
Hongmei Chen ${ }^{1}$, Zhifei Cui ${ }^{1}$, Yuanyuan Yan ${ }^{1}$, Zhan $\mathrm{Ma}^{1}$, Xiaole Gu${ }^{1}$, Yonghua $\mathrm{Bi}^{2}$
}

${ }^{1}$ Department of Ultrasound, Zhengzhou Central Hospital Affliated to Zhengzhou University, ${ }^{2}$ Department of Interventional Radiology, The First Affiliated Hospital of Zhengzhou University, Interventional Institute of Zhengzhou University, Interventional Therapy and Clinical Research Center of Henan Province, Zhengzhou, China

\section{To the Editor,}

We enjoyed reading the article entitled "The use of Doppler ultrasonography for pre- and post-surgery monitoring of abdominal aortic aneurysm. A case report" by Georgescu et al in the March 2016 issue of the journal [1]. The authors reported an interesting case of abdominal aorta aneurysm (AAA) monitored by Doppler Ultrasonography (US) for 3 years, and concluded that contrast enhancement US is reliable for investigating the complications of AAA after endovascular aneurysm repair (EVAR). Doppler US examination is a kind of reliable, reproducible and non-invasive technique, which should serve as the first choice to perform surveillance, such as prosthesis assessment and leakage detection after EVAR. However, the authors still needed CT scan to check the size and locations of the AAA before surgery. Interestingly, they performed a surveillance of the prosthesis not only by Doppler US but also by CT scan. We want to know whether Doppler US is capable of performing pre- and post-surgery monitoring of AAA without CT scan. As the authors pointed out, although contrast enhancement US can indicate the location of leak [2], these results must be interpreted with caution and the decision to perform second EVAR should be taken only corroborating with angiography [1].

Received 16.03.2016 Accepted 18.03.2016

Med Ultrason

2016, Vol. 18, No 2, 264, DOI: 10.11152/mu.2013.2066.182.dpp

Corresponding author: Prof Yuanyuan Yan, MD

Zhengzhou Central Hospital,

195 Tongbai St., Zhengzhou 450053, China.

Tel.: +860371 67692350 ;

Fax: +86037167692350

Email: 275547292@qq.com
In our department, preoperative examination and postoperative surveillance of AAA is usually performed by CT angiography, which is able to clearly show the entire aorta, the metallic prosthesis, and the type of endoleak in front or profile views. To the contrary, Doppler US is difficult to reveal the whole aorta in one screen, and metallic prosthesis is hard to see clearly due to the ultrasonic artifact of metal. Besides, this reported case also reveals the difficulty in determining the cause of leakage after EVAR by Doppler US monitoring [1]. In our opinions, the role of Doppler US and CT scan is complementary in AAA monitoring. Doppler US is a very useful preliminary screening technique for AAA before and after EVAR, due to the accurate examination of leak flow and avoidance of radiation exposure. CT scan is wanted to show the whole aorta and stent, as well as to confirm the type of endoleak. Besides, Doppler US will also play an important role in interventional operation, especially in the guidance of puncture, such as percutaneous puncture of portal vein of cavernous transformation, which is quite difficult under fluoroscopy.

Sources of Funding: This work was supported by the National Natural Science Foundation of China (Grant No. 81501569).

\section{Reference}

1. Georgescu ME, Arbeille P, Dobre M, Stefanescu V. The use of Doppler ultrasonography for pre- and post-surgery monitoring of abdominal aortic aneurysm. Case report. Med U1trason. 2016; 18: 127-130.

2. San Norberto EM, Taylor J, Vaquero C. Color-Doppler U1trasonography in the Monitoring of Endovascular Abdominal Aortic Aneurysm Repair. In: Grundmann R. (ed.). Diagnosis and Treatment of Abdominal and Thoracic Aortic Aneurysms Including the Ascending Aorta and the Aortic Arch. Rijeka, InTech 2011: 37-56. 


\section{Author's response}

\section{Monica Elia Georgescu' ${ }^{\text {, Philippe Arbeille }}{ }^{2}$, Michaela Dobre ${ }^{3}$, Victorita Stefanescu ${ }^{3}$}

${ }^{I}$ Centre Hospitalier Universitaire Trousseau, Tours, France, ${ }^{2}$ Department of Nuclear Medicine \& Ultrasounds, School of Medicine, University of Tours, France, ${ }^{3}$ Department of Morphological and Functional Sciences, Faculty of Medicine and Pharmacy, "Dunarea de Jos" University of Galati, Romania

First, we want to thank you for your interest in our article regarding the use of Doppler ultrasonography for pre- and post-surgery monitoring of abdominal aortic aneurysm (AAA).

Regarding our article, you asked whether Doppler US is capable of performing pre- and post-surgery monitoring of AAA without computerized tomography (CT). You also stated that the roles of Doppler US and CT scan are complementary in AAA monitoring. We are happy to address these concerns.

Regarding the ability of Doppler US to perform preand post-surgery monitoring of AAA, on the present case report we mention that the patient underwent CT scan, Angiography and contrast enhancement ultrasounds (CEUS). Doppler US is the usual technique used for the detecting of AAA as well as for pre-surgery monitoring, however the final decision to perform surgery is taken following CT scan examination. Currently, CEUS is considered more reliable than Doppler US for the discovery of the small leakages while monitoring the endovascular aneurysm/aortic repair EVAR [1,2], provided that the CEUS technique is performed by a trained team of specialists on highly performing equipment (making this technique more costly than Doppler US). Unlike of Doppler US, the CEUS technique allows accurate visualization of the metallic prosthesis, avoiding the ultrasonic artifact of metal. The conclusion from our case study is that CEUS provided information very close to those obtained through the CT scan. The purpose of the article is to demonstrate that CEUS is a more reliable technique than Doppler US for pre- and post-surgery monitoring of abdominal aortic aneurysm. We also mention in the article the limitation of the CEUS technique, and do not conclude, from this single case report, that the CEUS could replace $\mathrm{CT}$ and angiography.

Regarding the complementarity of Doppler US and CT scan in AAA monitoring, we agree to your comment. We consider that CEUS avoids potential aggravation of nephropathies caused by repeated ionizations, making it a suitable the alternative to CT scan in specific cases when patients already shows signs of renal failure. The conditions for the reliability of the CEUS method include top quality technical apparatus, highly trained and experienced experts collaborating with all teams involved in AAA treatment, as well as a good echogenicity of the patient (allowing the use of ultrasounds). However, we agree that CT scan examination is the only technique capable to display the aorta and the prosthesis in its entirety, as well as to establish the type of leakage at the level of the aneurismal sac.

\section{References:}

1. Abbas A, Hansrani V, Sedgwick N, Ghosh J, McCollum CN. 3D contrast enhanced ultrasound for detecting endoleak following endovascular aneurysm repair (EVAR). Eur J Vasc Endovasc Surg 2014; 47: 487-492.

2. Sarlon G, Lapierre F, Sarlon E, Bartoli MA, Magnan PE, Branchereau A. Surveillance des endoprothèses aortiques abdominales: intérêt de l'échographie-doppler standard et avec contraste. J Mal Vascul 2009; 34: 34-43. 